\title{
A retrospective study
}

\section{Yoshiaki Terao $^{1}$, Makito Oji ${ }^{1}$, Natsuko Oji1, Shigehiko Urabe ${ }^{1}$, Shozo Tominaga ${ }^{1}$, Maki Ono ${ }^{1}$, Makoto Fukusaki ${ }^{1}$, Tetsuya Hara². \\ 1, Department of Anesthesia, Nagasaki Rosai Hospital. \\ 2, Department of Anesthesiology, Nagasaki University School of Medicine.}

\begin{abstract}
Background: There is no gold standard pain management after total hip arthroplasty (THA). There are controversial reports whether lateral femoral cutaneous nerve-block (LFCNB) combined with opioid and NSAID is effective for postoperative pain after $T H A^{1,2)}$. This retrospective study was carried out to determine whether LFCN combined with femoral nerve-block (FNB) is effective for postoperative pain after THA.
\end{abstract}

Methods: The Institutional Research Committee approves this retrospective study and to waive informed consent because study procedure was routine examination after THA. This study was conducted in Nagasaki Rosai Hospital from June 2015 to November 2015 (pre-LFCNB period) and June 2016 to November 2016 (post-LFCNB period). We started LFCNB combined FNB in the all patients underwent THA before general anesthesia from April 2016. We included consecutive 39 patients underwent THA by the posterior approach without LFCNB combined with FNB (NB group) in pre-LFCNB period and 45 patients underwent THA by the posterior approach with LFCNB and FNB (B group) in postLFCNB period. Exclusion criteria were spinal anesthesia, allergy against local anesthetics, revision arthroplasty and bilateral arthroplasty. Ultrasound guided LFCNB and FNB with each $0.25 \%$ levobupivacaine $20 \mathrm{ml}$ were performed before general anesthesia in B group. Anesthesia was induced with thiamylal or propofol followed by sevoflurane, or total intravenous anesthesia with propofol. Fentanyl and remifentanil were used as the analgesic agent, and rocuronium was used as the neuromuscular blocking agent during anesthesia. $50 \mathrm{mg}$ of flurbiprofen or $1000 \mathrm{mg}$ of acetaminophen was administrated intravenously before skin closure. The pain score was evaluated by numeric rating scale (NRS) from 0 to 10 at 0 (T0), 1 (T1), 3 (T2), 6 (T3) and 9 (T4) hour after surgery, and in the next morning (TN). The patients were administrated diclofenac sodium, loxoprofen or pentazosine for postoperative pain, if needed. We evaluated the number of administration of them until 12 hour and 24 hour after surgery. Surgeons evaluated the satisfaction degree of postoperative pain managements by NRS from 1 to 5 in the one postoperative day. The results are expressed as median (IQR). Statistical analyses were performed by Mann-Whitney $U$ test or Chi square test. A $p$ value $<0.05$ was considered statistically significant.

Results: Height, weight and number of male out of patient's characteristics were more in NB group compared with B group (Table 1). Operative time, anesthetic time, the amount operative blood loss, the amount of fentanyl and the frequency of TIVA in B group were more than those in NB group (Table 2). NRS scores for postoperative pain at T0 $[0(0,4)$ vs $5(0,8)]$, T1 $[0(0,2)$ vs $5(2$, $7)]$, T2 [1 $(0,2)$ vs $4(2,5)]$, T3 $[1(0,3)$ vs $3(2,4)]$ and T4 [2 $(0,4)$ vs $3(0,5)$ ] were lower in B group compared with NB group (Figure). There are no significant differences in the frequency of analgesics between two groups. NRS score for satisfaction in B group was superior to NB group (Table 3).

Conclusion: LFCN combined with FNB is effective for postoperative pain management during early postoperative period after THA.

\section{References:}

1, Acta Anaesthesiol Scand 2016; 60: 1297-305.

2, BMC Anesthesiol 2016; 16: 21.

Acknowledgement: This research was supported, in part, by research funds to promote the hospital functions of the Japan Labor Health and Welfare Organization.

Competing Interests: We have no competing interests.
Table 1. Patient characteristics

\begin{tabular}{lccr}
\hline Group & NB $(39)$ & $\mathrm{B}(45)$ & $\mathrm{p}$ \\
\hline Age $(\mathrm{yr})$ & $66(61,70)$ & $68(61,79)$ & 0.17 \\
Height $(\mathrm{cm})$ & $155(152,160)$ & $151(147,156)$ & $<0.01$ \\
Weight $(\mathrm{kg})$ & $60.1(52.3,70.0)$ & $53.0(47.5,62.3)$ & 0.01 \\
BMI & $25.1(23.2,26.7)$ & $22.9(21.5,25.9)$ & 0.12 \\
Male & $14 / 39$ & $6 / 45$ & 0.02 \\
Right & $17 / 39$ & $27 / 45$ & 0.13 \\
Hypertension & $21 / 39$ & $23 / 45$ & 0.80 \\
Diabetes & $6 / 39$ & $7 / 45$ & 0.98 \\
\hline
\end{tabular}

Table 2. Operative variables

\begin{tabular}{lllr}
\hline Group & NB (39) & B (45) & \multicolumn{1}{l}{$\mathrm{p}$} \\
\hline Operative time (min) & $51(49,62)$ & $75(64,93)$ & $<0.01$ \\
Anesthetic time (min) & $109(102,121)$ & $131(120,149)$ & $<0.01$ \\
TIVA: Sevoflurane & $16: 23$ & $36: 9$ & $<0.01$ \\
Blood loss $(\mathrm{g})$ & $120(93,200)$ & $170(130,245)$ & 0.02 \\
Fentanyl (mL) & $5(5,7)$ & $10(7,10)$ & $<0.01$ \\
Remifentanyl (mcrg/kg) & $5.8(3.1,8.6)$ & $5.1(3.6,9.1)$ & 0.56 \\
Flur: Aceta & $38: 1$ & $40: 5$ & 0.12 \\
\hline
\end{tabular}

Flur:Aceta; flurbiprofen:acetaminophen

Figure. Individual numeric rating scale during study period.

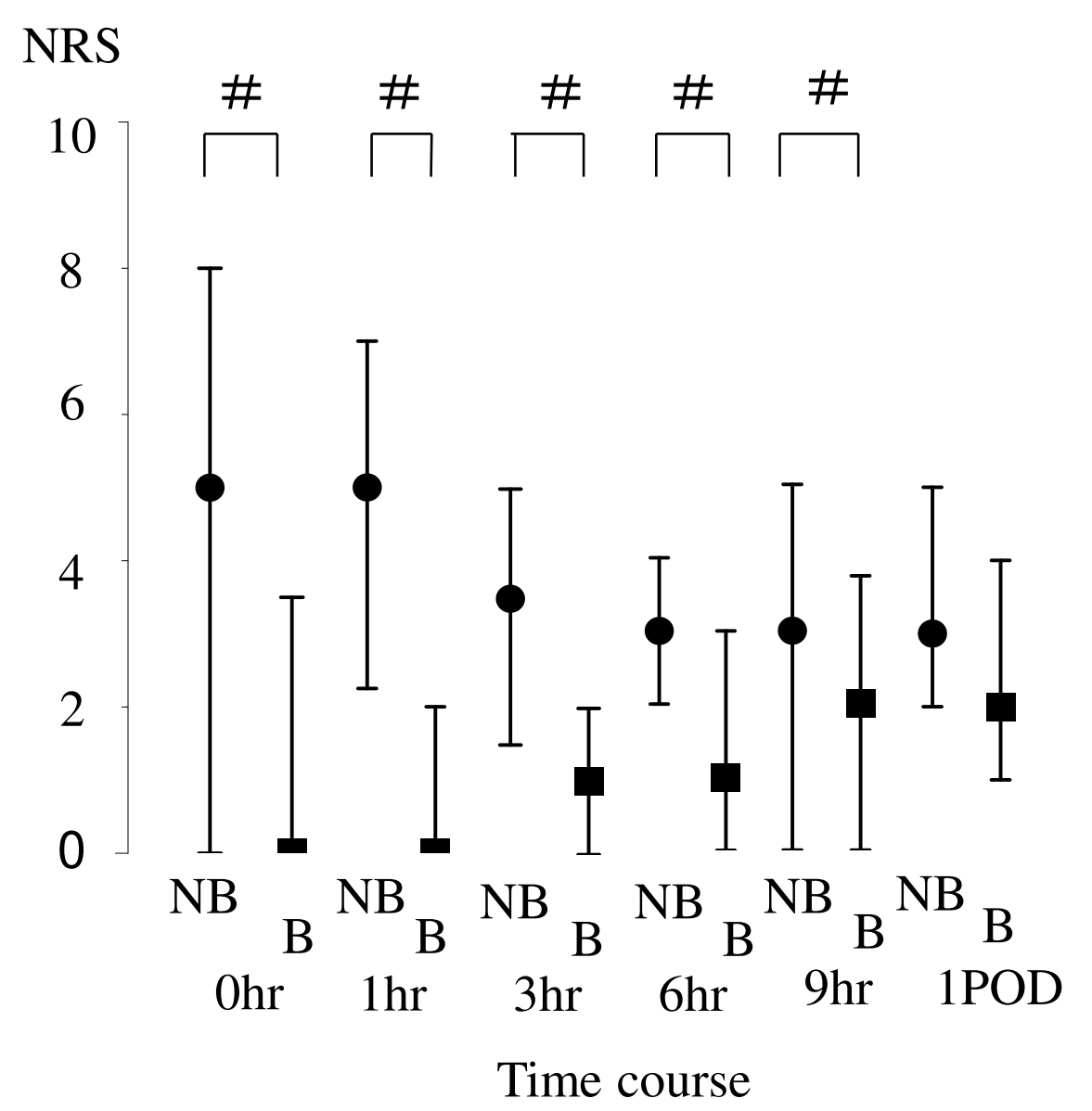

NB, no nerve block; $B$, femoral and lateral femoral cutaneous nerve block

Table 3. Postoperative variables

\begin{tabular}{llll}
\hline Group & NB $(39)$ & B $(45)$ & $\mathrm{p}$ \\
\hline Blood loss $(\mathrm{g})$ & $110(70,193)$ & $130(70,173)$ & 0.92 \\
PONV & $9 / 39$ & $16 / 45$ & 0.21 \\
analgesics frequency-12hr & $1(0,1)$ & $1(0,1)$ & 0.66 \\
analgesics frequency-24hr & $2(1,3)$ & $2(1,2)$ & 0.17 \\
Satisfaction NRS & $4(2,4)$ & $2(2,3)$ & $<0.01$ \\
\hline
\end{tabular}

PONV, postoperative nausea and vomiting 